\title{
In Memory of Marina Ratner 1938-2017
}

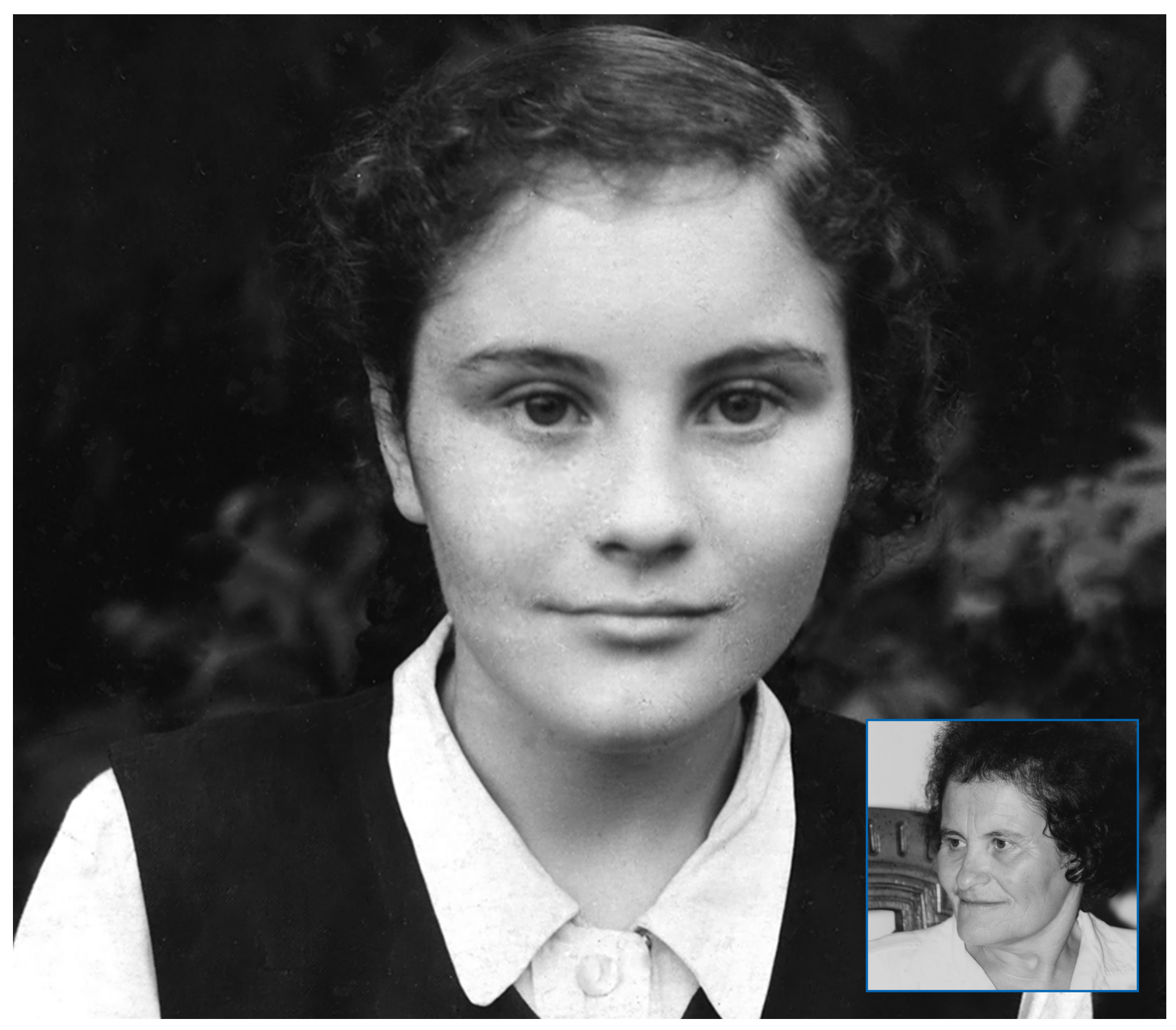

For permission to reprint this article, please contact: reprint -permission@ams.org.

DOI: http://dx.doi.org/10.1090/noti1828 


\section{Marina Ratner}

\section{S. G. Dani}

This article has been reproduced with permission from Bhāvanā, October 2017, Vol. 1, Issue 4. See bhavana.org . in/marina-ratner-1938-2017.

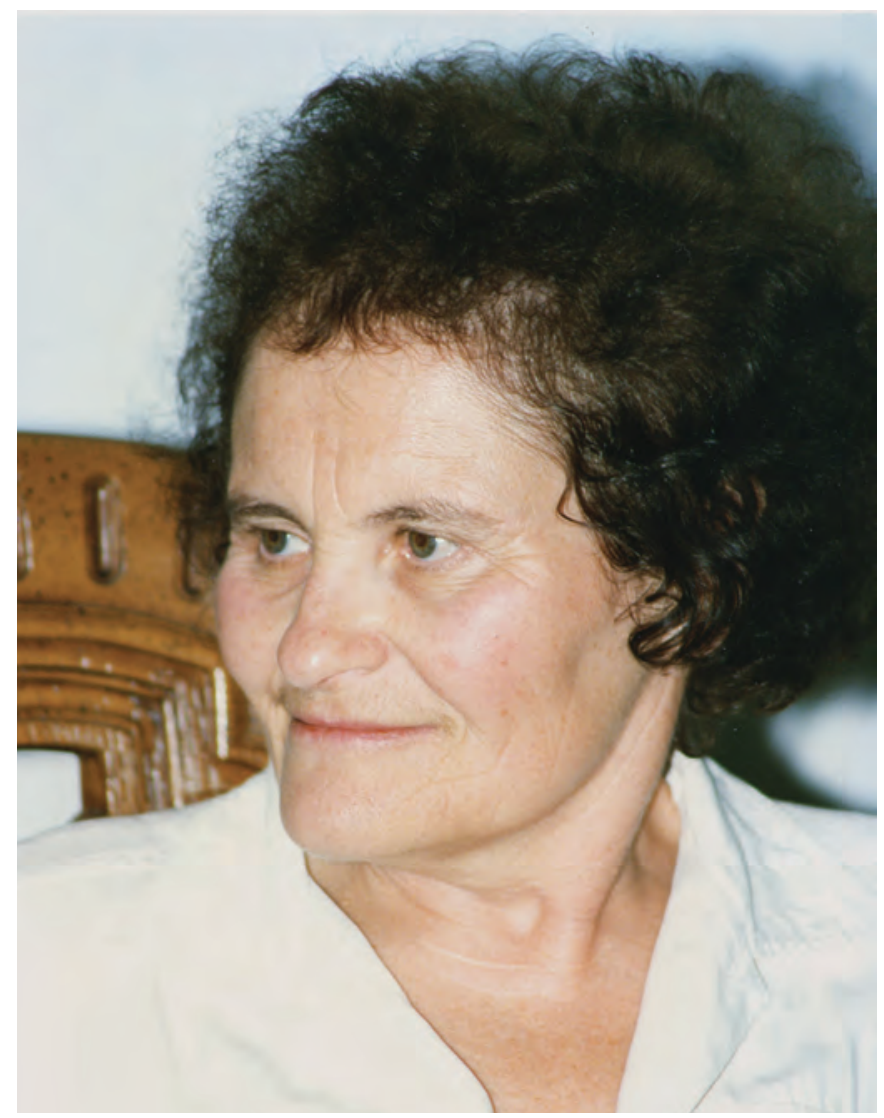

S. G. Dani was affiliated with the Tata Institute of Fundamental Research (TIFR), Mumbai for over four decades, until mandatory retirement in 2012. Subsequently, he was associated with IIT Bombay and, more recently, is with the UM-DAE Centre for Excellence in Basic Sciences, Mumbai, a collaborative endeavor of the University of Mumbai and the Department of Atomic Emergy of the Government of India. His email address is shrigodani@gmai1. com.

For permission to reprint this article, please contact: reprint -permission@ams.org.

DOI: http://dx.doi.org/10.1090/noti1835
Marina Evseevna Ratner, renowned for her work in dynamics, passed away on July 7, 2017, at her home at El Cerrito, California, USA, at the age of 78 . Her profound contributions, establishing the Raghunathan conjecture and its variants, from the 1990s when she was in her early fifties, have become a milestone in homogeneous dynamics and have had an impact on the study of a broad range of areas of mathematics, including dynamics, diophantine approximation, ergodic theory, geometry, and Lie group theory.

Marina was born in Moscow on October 30, 1938 to scientist parents, her father a plant physiologist and mother a chemist. As a Jewish family they had a difficult time in Russia at that time. In particular, her own mother lost her job for having corresponded with none other than her mother who was in Israel, which was considered an enemy state. Marina was educated in Moscow and fell in love with mathematics when she was in the fifth grade; "mathematics came naturally to me and I felt unmatched satisfaction solving difficult problems" she was to aver later. ${ }^{1}$ After completing school she gained admission to the Moscow State University, which, with the dawning of the Khrushchev era, had begun to accept Jewish students on an equal footing.

After graduating from the University in 1961, Ratner worked for four years as an assistant in the Applied Statistics Group of A. N. Kolmogorov, the celebrated Russian mathematician who laid the foundations of measure-theoretic probability theory and had a great influence on her during her undergraduate years. Kolmogorov had an intensive training program for talented high school students with which Marina was actively involved. It is also during these years that she gave birth to a daughter from a short-lived marriage.

\footnotetext{
${ }^{1}$ M. Cook, Marina Ratner, in: Mathematicians: An Outer View of the Inner World, Princeton University Press, 2009, pp. 90-91.
} 
In 1965 Marina took up research under the supervision of Ya. G. Sinai, a former student of Kolmogorov, renowned, in particular, for his role jointly with his advisor in the development of the very influential "entropy" invariant in ergodic theory around $1960 .{ }^{2}$ In the context of how the theory was developing then, in Russia, the geodesic flows associated with surfaces of negative curvature had emerged as crucial examples for study from an ergodic-theoretic point of view, and Ratner also wrote her thesis on this topic. ${ }^{3}$ Apart from the examples themselves, a general class of systems known as Anosov flows, named after D. $\mathrm{V}$. Anosov who introduced and proved some deep results about them, were of interest, and Ratner worked on the asymptotic statistical properties of these flows as well. For the work she received the equivalent of the $\mathrm{PhD}$ degree in 1969 from Moscow State University.

After receiving the degree Ratner was employed as an assistant at the High Technical Engineering School in Moscow. In 1970 the government of USSR was led, in the face of international pressure, to increase substantially the emigration quotas, sparking an exodus of Russian Jews to Israel, of which earlier there had just been a trickle. Notwithstanding the relaxation in the policy, the government and the bureaucracy in general were highly resistant to emigration and treated those desirous of migrating with utmost severity in various ways. Thus, when Ratner applied for a visa that year (1970) to emigrate to Israel, she was dismissed from the job at the Engineering School.

\footnotetext{
${ }^{2} \mathrm{~A}$ dynamical system in the present context means a one-parameter group of transformations of a space (sometimes called the phase space), with the parameter representing time (which could be continuous or discrete); the theory focuses on the long-term behavior of the trajectories of points under application of the transformations, namely, as the time parameter tends to infinity. In ergodic theory the phase space is further considered equipped with a measure of unit mass, namely, a probability is associated for points to belong to various subsets; the system is said to be measure-preserving if the probability remains unchanged when any of the transformations under consideration is applied to a point. In these instances one often focuses on trajectories of "generic points" in terms of the measure or equivalently in statistical terms with respect to the initial point. The Kolmogorov-Sinai entropy is a nonnegative number associated with each measure-preserving system, and when the entropy of two systems is different, their long-term behavior is different. The invariant thus enabled distinguishing dynamical systems on a much finer scale than was possible before.

${ }^{3}$ The "geodesic flow" consists of starting with a given point on the surface (or manifold in general) and a direction from that point and moving on, along the distance-minimizing paths corresponding to the geometry of the surface, for the desired amount of time, and noting the point of arrival and the direction of movement at that point; thus, the phase space in this case is formed of pairs consisting of a point of the surface and a direction at that point, and the above procedure describes how the transformation is defined. On the usual sphere the trajectories (paths) of such a flow would follow the great circle, and return to the original point after a fixed amount of time. However, when the surface has negative curvature, the trajectories move away from each other substantially, exponentially in time, and when the surface is compact, "almost all" of them (statistically) tend to fill up the whole space with the passage of more and more time.
}

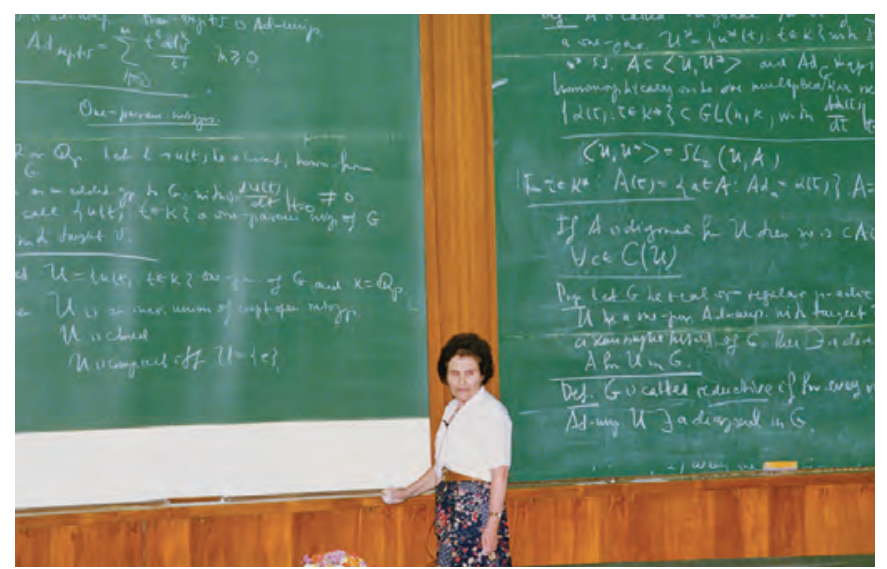

Marina Ratner giving a talk at the International Colloquium at TIFR, 1996.

Ratner landed in Israel, with her daughter, in 1971 and served as a lecturer until 1974 at the Hebrew University of Jerusalem and then at the Pre-academic School of the Hebrew University for another year. During this period Ratner continued her work on the geodesic flows, and also their generalizations in higher dimensions, and established in particular a property manifesting complete randomness of behavior of the trajectories of the Anosov flows, known as the Bernoulli property, that was much sought after in various systems.

In the West the study of flows analogous to Anosov flows, called "Axiom A" flows, was introduced by Stephen Smale, then at the University of California (UC), Berkeley. Apart from a certain generality of setting, this study involves separating the role of the measure and understanding the dynamics in terms of the construction of special kinds of partitions of the space, known as Markov partitions. Profound work was done in this direction by one of Smales's students, Robert E. Bowen, known commonly by his adopted name "Rufus" Bowen; the work led Bowen to the construction of invariant measures inherently associated to the systems in more general settings, now known as Bowen measures. Bowen completed his doctorate in 1970 and joined the Berkeley faculty in the same year. Not surprisingly, Bowen was interested in the work of Marina Ratner, and their correspondence during her Jerusalem years culminated in Ratner getting an invitation from UC Berkeley, which she joined in 1975 as acting assistant professor.

Another class of flows, called horocycle flows, are seen to have become a major love for Marina after moving to Berkeley. The geodesic flow associated with a surface of constant negative curvature has two natural companion flows, called the contracting horocycle flow and the expanding horocycle flow; they are actually twins, interchangeable through time reversal of directions at each point, so one may simply talk of the horocycle flow. Passing through each point of the phase space (consisting of a point of the surface together 
with a direction at the point), there is a uniquely defined curve such that if we pick two points on any one of these curves and consider their trajectories under the geodesic flow we find them getting closer and closer with the passage of time, with the distance between the corresponding points of the trajectories tending to zero. Moreover, there is a natural parametrization on these curves with respect to which they can be thought of as the trajectories of a measure-preserving flow, and that is the (contracting) horocycle flow associated with the surface; the expanding horocycle flow arises similarly from consideration of trajectories of the geodesic flow in the reverse direction. These flows have historically proved to be very useful in studying the properties of the geodesic flows. While in the nature of things the horocycle flow would seem just a sidekick of the geometrically majestic geodesic flow, in the theory of dynamical systems the former has acquired a stature of its own, on account of some of its unique properties.

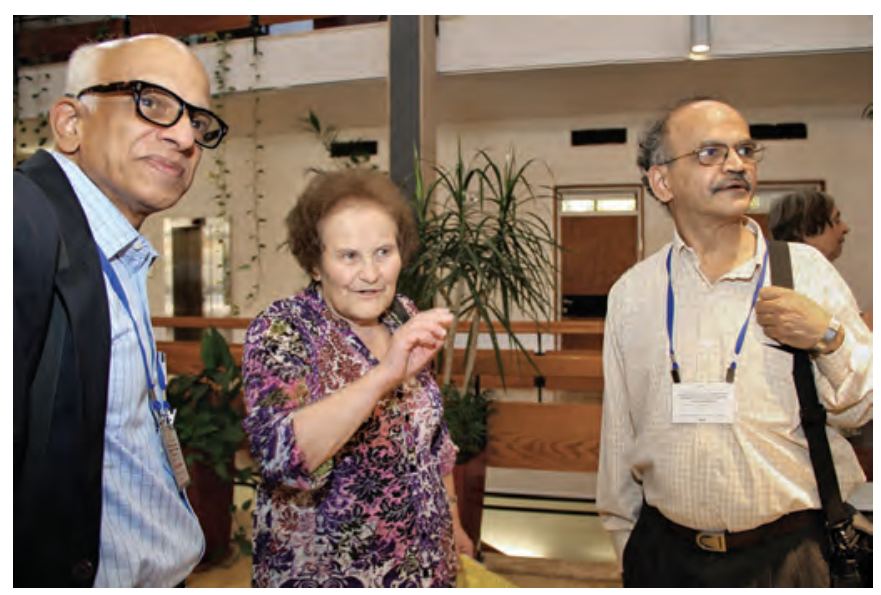

Marina Ratner with M. S. Raghunathan and S. G. Dani at the conference held in her honor at the Hebrew University of Jerusalem in October 2013.

In two papers published in 1978 and 1979 Ratner showed that the horocycle flows are "loosely Bernoulli" while their Cartesian squares are not "loosely Bernoulli"; the loose Bernoullicity property was introduced by J. Feldman, a colleague at Berkeley, and concerns the flow being similar to the standard winding line flows on the torus along lines with irrational slopes, if one allows the time parameter associated with the trajectories to be modified suitably. The fact, as established by Ratner, that the Cartesian square is not loosely Bernoulli for the horocycle flows is rather curious and was the first such instance to be found.

The early 1980s saw a major breakthrough in the understanding of the horocycle flows associated with compact surfaces, of constant negative curvature, at the hands of Ratner. A major question involved was the following: given two such surfaces whether the horocycle flows associated with them being isomorphic to each other as measure-pre- serving flows would imply that the surfaces themselves are geometrically indistinguishable. The answer to the corresponding question in the case of the geodesic flows is a definitive no since in fact the flows being Bernoullian means (by a well-known result of D. S. Ornstein) that any two of them are isomorphic to each other (up to a rescaling of the time parameter), irrespective of the specific geometries of the underlying surfaces. Ratner proved that on the other hand the horocycle flows corresponding to two distinct compact surfaces of constant negative curvature would never be isomorphic. This kind of phenomenon is referred to as rigidity. She also exhibited various variations of the rigidity property of the horocycle flows, through a series of papers, describing their factors, joinings, etc. (joining is a technical construction that enables comparing two systems with regard to the nature of their dynamics). Two of the three papers in this respect appeared, in 1982 and 1983, in the Annals of Mathematics. Apart from the immediate outcomes, which were striking in themselves, the work has germs of the ideas involved in the later celebrated work on the Raghunathan conjecture.

Let me now come to the Raghunathan conjecture, resolution of which was the major feat of Marina's work. Genesis of the conjecture is intricately connected with my student years at the Tata Institute of Fundamental Research, and it would be worthwhile to recall some details in that regard. I did my doctoral work in the early 1970s under the supervision of M. S. Raghunathan on flows on homogeneous spaces. ${ }^{4}$ The thesis dealt primarily with the Kolmogorov property, which is a statistical property concerning a strong form of mixing, with no direct bearing on the behavior of individual orbits. However, in a paper written shortly after completing the thesis paper (before the award of the degree, in fact) I proved that all the orbits of actions of a class of flows, more specifically horospherical flows, are dense in the space. Around that time Jyotsna Dani (my wife) who was working under the supervision of S. Raghavan, at TIFR, had proved that for any vector whose coordinates are nonzero and not rational multiples of each other, the

\footnotetext{
${ }^{4}$ For an idea of homogeneous spaces and dynamics on them let us consider a Euclidean space and agree to identify two given vectors of the space if their difference has integer coordinates, namely, we view the vectors modulo the lattice of vectors with integral coordinates; geometrically in effect we are considering a torus, and translations by vectors on any particular line define a translation flow on the torus. Similarly, when the elements of various matrix groups, more generally Lie groups, are considered modulo elements of large enough discrete subgroups (called lattices) we get what are called homogeneous spaces with a natural finite measure on them, and matrix multiplication by elements from a one-parameter subgroup of the ambient group, considered modulo the lattice, defines a flow on the homogeneous space. In the particular case when the group involved is the "modular group," the group of $2 \times 2$ matrices with real entries and determinant 1 , the flows arising in this way (other than those which are periodic) in fact correspond to the geodesic and horocycle flows associated with various surfaces with constant negative curvature, via certain natural identification of the phase space of the flow with a homogeneous space of the modular group.
} 


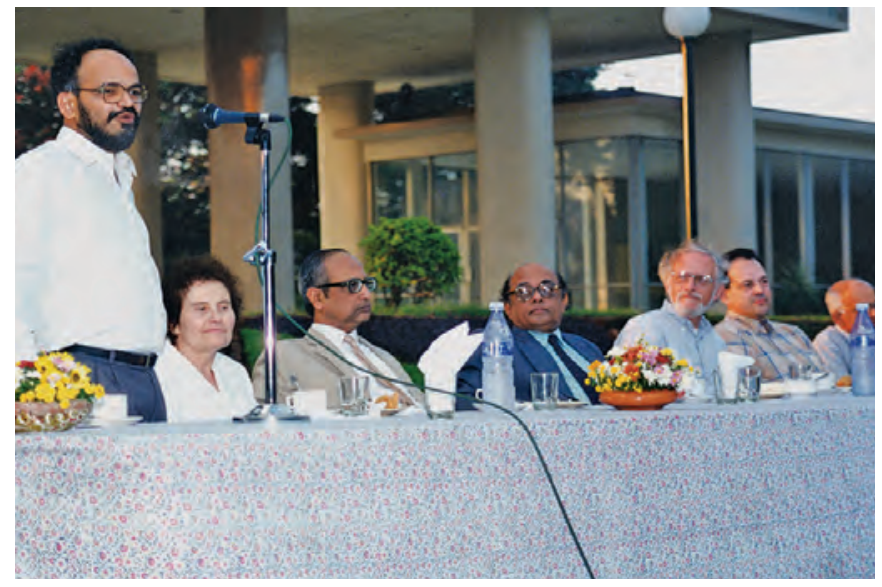

Marina Ratner at the inaugural function of the

International Colloquium on Lie Groups and ErgodicTheory at theTata Institute of Fundamental Research, Mumbai, 1996. The others on the dais, from left to right are, S. G. Dani, Virendra Singh, R. Chidambaram, Hillel Furstenberg, Anatole Katok, and M. S. Raghunathan.

orbit under the action of the group of integral unimodular (determinant 1) matrices on the corresponding Euclidean space, is dense in the Euclidean space. At some point in time around 1975, which had these events in the background, when I was talking to Raghunathan about possible problems to pursue, he casually suggested a statement on the behavior of what are called unipotent flows ${ }^{5}$ and quite nonchalantly added "call it my conjecture and prove it." He pointed out that proving it would in particular settle the conjecture of Oppenheim on density of values of indefinite forms at integral points, ${ }^{6}$ which was one of the hallowed problems at that time in the Tata Institute precincts.

That statement of Raghunathan-the Raghunathan conjecture-first recorded in print in my Inventiones Mathematicae (1981) paper, is that the closure of any orbit of a unipotent one-parameter subgroup acting on a homogeneous space of finite volume is the orbit of a (possibly larger) subgroup of the ambient Lie group; in particular this means that each of these closures of orbits

\footnotetext{
${ }^{5}$ A flow on a homogeneous space of a matrix group as in the previous footnote is said to be unipotent when the one-parameter group involved consists of unipotent matrices, namely, matrices that have no eigenvalue, even in complex numbers, other than 1; for a general Lie group there is a variation of this involved. In the case of the modular group there are precisely the horocycle flows associated with surfaces of constant negative curvature and finite area.

${ }^{6}$ The conjecture originating from a paper of Alexander Oppenheim from 1929 predicted that for any nondegenerate indefinite quadratic form in at least three variables, which is not a multiple of a form with rational coefficients, the set of its values at integer tuples is dense in real numbers. It had been worked on by several notable number theorists, and by the $1980 \mathrm{~s}$ many partial results were known, confirming the conjecture under various restrictions, but a general solution had eluded the efforts.
}

is a geometrically nice object-this is a remarkable thing to happen for a dynamical system, the crucial point being that the statement is being made for every orbit and not only the generic ones. ${ }^{7}$ In that paper I proposed another conjecture, as a step toward proving the Raghunathan conjecture, relating to measures that are invariant under these flows, namely, that the ergodic ${ }^{8}$ ones from among them in fact arise as measures invariant under the action the larger subgroups as above and are supported on a single orbit of the subgroup; a weak result was proved in the paper in that direction, partially vindicating the conjecture. Ratner proved the latter conjecture, which she referred to as "Raghunathan's measure conjecture," and in a separate paper deduced the original topological version. ${ }^{9}$

The Oppenheim conjecture itself, which had inspired the Raghunathan conjecture, was settled by G. A. Margulis in 1986 by proving a much weaker statement than the Raghunathan conjecture but in a similar spirit. Not surprisingly, proving the full conjecture led to a much broader perspective in the study of values of quadratic forms at points with integer coordinates, and many other applications, some quite immediately and many more over the years. There have been numerous results since then making use of Ratner's theorems in crucial ways, in a variety of contexts, and there is no doubt that it will serve as a mainstay for a good deal of mathematics in the coming decades.

The proofs are long and intricate and involve various ancilliary results. However, there is a beautiful key idea that concerns observing and adopting a property of the unipotent flows, which it may be worthwhile to recall. It may be informally stated as the following: if you find two trajectories of the flow having stayed quite close for reasonably long, then you can expect them to stay fairly close for substantially longer. This property of the unipotent flows, now called the Ratner property, has since acquired significance as a dynamical phenomenon.

As to be expected, Ratner gained considerable professional recognition. While her initial appointment at Berkeley had been a source of some controversy in the Department, her subsequent rise in the ranks seems to have

\footnotetext{
${ }^{7}$ For a one-parameter flow the orbit of a point is the set of all the points that can be reached by application of one of the transformations from the flow (including those corresponding to the negative value of the time parameter); similar terminology applies also to a more general group of transformations, in place of the one-parameter flows. The closure of the orbit means all the points that can be approximated by points on the orbit. In a typical dynam$i c a l$ system, even when the closures of almost all orbits are the whole space, for others, the exceptional ones, the closures can be very crazy. For instance, for the geodesic flows as in the above discussion there are orbit closures whose intersection with some curves transversal to the flow consists of a mess of uncountably many individual points disconnected from each other.

${ }^{8}$ Those that cannot be expressed nontrivially as a sum of two invariant measures.

${ }^{9}$ During the interim there were various partial results proved in that direction, but we shall not concern ourselves with it here.
} 
been smooth-sailing. She was elected in 1992 to the American Academy of Arts and Sciences, and in 1993 she was awarded the Ostrowski Prize. ${ }^{10}$ In 1994 she won the John J. Carty Prize of the National Academy of Science. She was invited as a plenary speaker at the International Congress of Mathematicians, held in Zurich, in 1994, to become only the third woman mathematician, along with Ingrid Daubechies, to receive such an honor; Emmy Noether (in 1932) and Karen Uhlenbeck (in 1990) are the two women to have received the distinction earlier.

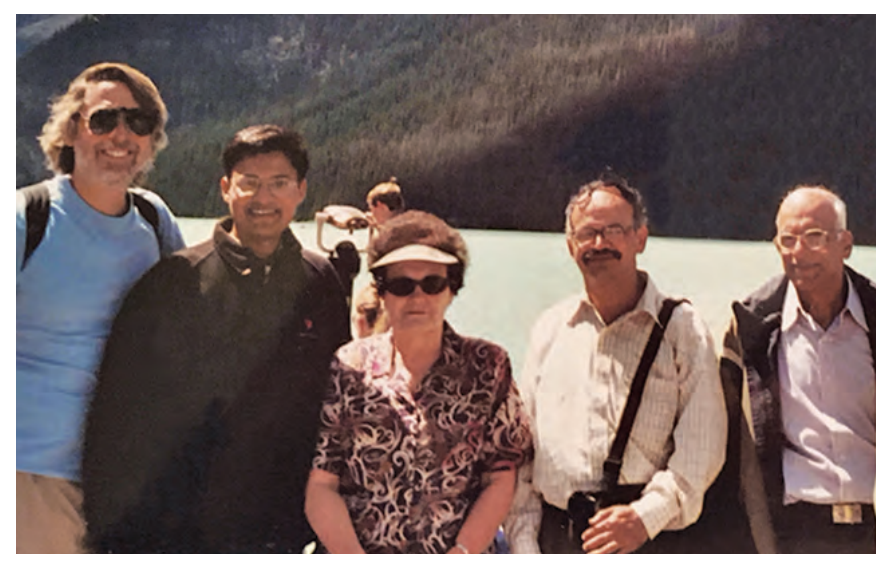

Marina Ratner at an excursion to Lake Louise, Alberta, Canada, with some of the delegates to the Conference on ErgodicTheory, held at the Banff International Research Station, Banff, Alberta, Canada, in July 2005. In the photo, from left to right, are DaveWitte Morris, Nimish Shah, Marina Ratner, S. G. Dani, and M. S. Raghunathan.

A conference on "Homogeneous Dynamics, Unipotent Flows, and Applications" was held at the Hebrew University of Jerusalem, October 13-17, 2013, in honor of Marina Ratner and her work, hosted by the Israel Institute for Advanced Studies and supported by the European Research Council. Earlier that year the Hebrew University of Jerusalem conferred upon her an honorary doctorate, at its Convocation held on June 16, 2013.

My personal contacts with Marina were, unfortunately, only sporadic, though they extended over a stretch of more than three decades. ${ }^{11}$ I found her a very warm-hearted person, going out of her way to extend hospitality, which I had numerous occasions of enjoying together with my family.

\footnotetext{
$\overline{{ }^{10} \text { The prize is awarded, since 1989, by the Ostrowski Foundation every }}$ alternate year for outstanding achievements in pure mathematics or foundations of numerical analysis.

${ }^{11}$ The first of these was in the spring of 1982 when I had an opportunity to visit the University of California, Berkeley for the semester; though in anticipation of the visit I was hoping for a serious mathematical interaction with her, it turned out, much to my disappointment, that she was on sabbatical leave during the period, which she was spending at Stanford University, and we happened to meet only occasionally during her brief visits to Berkeley.
}

We had an International Colloquium on Lie Groups and Ergodic Theory at TIFR in 1996 and the pleasure of having Ratner as one of the speakers. She also contributed a paper to the proceedings of the colloquium on $p$-adic and S-arithmetic generalizations of the Raghunathan conjecture.

My last meeting with her was in 2015, when there was a special semester organized at the Mathematical Sciences Research Institute, Berkeley, on homogeneous dynamics. On one evening she had organized a dessert party at her home. It had been a wonderful evening thanks, apart from the sumptuous desserts of wide variety, to the warm reception by Marina that she conducted so cheerfully and energetically. My wife and I got to see some photographs from her visit to Mumbai, which she had dug out for the occasion. We also got to meet her daughter Anna and her children. On receiving the news of her sad demise, I emailed Anna a condolence message expressing shock and sadness, in which I also mentioned how energetic Marina had seemed at the party. In her response Anna added, "This is all very sudden and unexpected and difficult to comprehend. She was always so full of energy." Indeed, her sad demise was very abrupt, and we deeply miss her lively presence amongst us.

\section{Credits}

Opening photo of Marina Ratner is courtesy of Anna Ratner. Photos of Marina Ratner at the 1996 International Colloquium at TIFR are courtesy of TIFR Archives.

Photo of Marina Ratner at the Hebrew University of Jerusalem, 2013 is courtesy of Israel Institute for Advanced Studies, The Hebrew University of Jerusalem.

Photo of Marina Ratner at Lake Louise is courtesy of Nimish A. Shah. 


\section{Ratner's Work on Unipotent Flows and Its Impact}

\section{Elon Lindenstrauss, Peter Sarnak, and Amie Wilkinson}

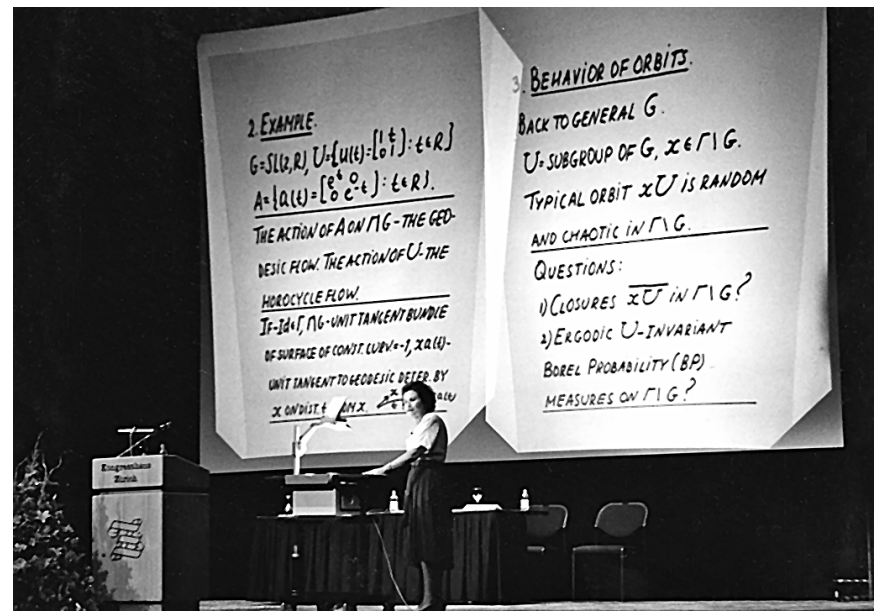

Ratner presenting her rigidity theorems in a plenary address to the 1994 ICM, Zurich.

In this note we delve a bit more into Ratner's rigidity theorems for unipotent flows and highlight some of their striking applications, expanding on the outline presented by

Elon Lindenstrauss is Alice Kusiel and Kurt Vorreuter professor of mathematics at The Hebrew University of Jerusalem. His email address is e1on@math .huji.ac . 71 .

Peter Sarnak is Eugene Higgins professor of mathematics at Princeton University and also a professor at the Institute for Advanced Study. His email address is sarnak@math.princeton.edu.

Amie Wilkinson is a professor of mathematics at the University of Chicago. Her email address is wi $1 \mathrm{kinso@math.} \mathrm{uchicago.edu.}$

For permission to reprint this article, please contact:

reprint-permission@ams.org.

DOI: https://doi.org/10.1090/noti/1829
Dani above. As the name suggests, these theorems assert that the closures, as well as related features, of the orbits of such flows are very restricted (rigid). As such they provide a fundamental and powerful tool for problems connected with these flows. The brilliant techniques that Ratner introduced and developed in establishing this rigidity have been the blueprint for similar rigidity theorems that have been proved more recently in other contexts.

We begin by describing the setup for the group of $d \times d$ matrices with real entries and determinant equal to $1-$ that is, $\operatorname{SL}(d, \mathbb{R})$. An element $g \in \operatorname{SL}(d, \mathbb{R})$ is unipotent if $g-1$ is a nilpotent matrix (we use 1 to denote the identity element in $G$ ), and we will say a group $U<G$ is unipotent if every element of $U$ is unipotent. Connected unipotent subgroups of $S L(d, \mathbb{R})$, in particular one-parameter unipotent subgroups, are basic objects in Ratner's work. A unipotent group is said to be a one-parameter unipotent group if there is a surjective homomorphism defined by polynomials from the additive group of real numbers onto the group; for instance

$$
u(t)=\left(\begin{array}{ll}
1 & t \\
& 1
\end{array}\right) \quad \text { and } \quad u(t)=\left(\begin{array}{ccc}
1 & t & t^{2} / 2 \\
& 1 & t \\
& & 1
\end{array}\right)
$$

In both cases it is easy to verify directly that these polynomials do indeed define a homomorphism: i.e., for any $s, t \in \mathbb{R}$ it holds that $u(t+s)=u(t) \cdot u(s)$. While there is essentially no loss of generality in discussing only the case of $\operatorname{SL}(d, \mathbb{R})$, a more natural context is that of linear algebraic groups - subvarieties of $\operatorname{SL}(d, \mathbb{R})$ defined 
by polynomial equations that are closed under multiplications and taking inverses (this notion actually makes sense for more general fields than the real numbers; if we want to emphasize that we are working with the field of real numbers we will call such groups linear algebraic groups over $\mathbb{R})$. Connected unipotent subgroups of $\operatorname{SL}(d, \mathbb{R})$ are always linear algebraic groups. Another nice class of examples are the orthogonal groups. Given a quadratic form $Q(\mathbf{x})$ over $\mathbb{R}$ (positive definite or not) in $d$ variables, one can consider the group $\mathrm{SO}(Q)$ of all matrices in $\operatorname{SL}(d, \mathbb{R})$ that preserve this form, i.e. $d \times d$-matrices $M$ so that $Q(M \mathbf{x})$ $=Q(\mathbf{x})$ for all $x \in \mathbb{R}^{d}$. This group will be compact if and only if $Q$ is a positive definite or a negative definite form.

Ratner's theorems on rigidity of unipotent group actions deal with the action of a unipotent group $U$ on a quotient space of $G$ by a discrete subgroup. An important example of such a quotient space is when $G=\operatorname{SL}(d, \mathbb{R})$ and $\Gamma=\operatorname{SL}(d, \mathbb{Z})$, in which case $G / \Gamma$ can be identified with the space lattices in $\mathbb{R}^{d}$ that have unit covolume. A lattice in $\mathbb{R}^{d}$ can be specified by giving $d$ linearly independent vectors that generate it - i.e. vectors $v_{1}, \ldots, v_{d} \in \mathbb{R}^{d}$ (that we prefer to think of as column vectors) so that $\Lambda=\mathbb{Z} v_{1}+$ $\cdots+\mathbb{Z} v_{d}$, and the condition that the lattice has unit covolume amounts to requiring that $\operatorname{det}\left(v_{1}, \ldots, v_{d}\right)=1$, or in other words that the matrix $g=\left(v_{1}, \ldots, v_{d}\right)$ obtained by joining together these $d$ vectors be in $\operatorname{SL}(d, \mathbb{R})$. The generators of the lattice $\Lambda$ are not uniquely determined: $v_{1}^{\prime}, \ldots, v_{d}^{\prime}$ generate the same lattice as $v_{1}, \ldots, v_{d}$ if and only if $\left(v_{1}^{\prime}, \ldots, v_{d}^{\prime}\right)=\left(v_{1}, \ldots, v_{d}\right) \gamma$ for $\gamma \in \operatorname{SL}(d, \mathbb{Z})$, in other words, lattices of unit covolume in $\mathbb{R}^{d}$ are in one-toone correspondence with elements of $\operatorname{SL}(d, \mathbb{R}) / \operatorname{SL}(d, \mathbb{Z})$. Any matrix $h \in \mathrm{SL}(d, \mathbb{R})$ acts on this space by left multiplication; in terms of lattices this amounts to the map from the space of unit covolume lattices to itself taking a lattice $\Lambda<\mathbb{R}^{d}$ to the lattice $\{h . v: v \in \Lambda\}$.

This quotient space has the important property of having finite volume, or more precisely an $\operatorname{SL}(d, \mathbb{R})$-invariant probability measure. A subgroup $\Gamma$ of a topological group $G$ which is discrete and such that $G / \Gamma$ has finite volume is called a lattice (admittedly, this can be a bit confusing at first since our basic example of such $G / \Gamma$ is the space of lattices in $\mathbb{R}^{d} \ldots$, though this terminology is consistent). Hermann Minkowski seems to have been the first to realize the importance of such quotients, and in particular the space of lattices in $\mathbb{R}^{d}$, to number theory at the turn of the 19th century. In the introduction to his book Geometrie der Zahlen, Minkowski writes ${ }^{1}$

This book contains a new kind of applications of analysis of the infinite to the theory of numbers or, better, creates a new bond between these two areas.. Geometry

\footnotetext{
${ }^{1}$ Translated from the original German to English.
}

of Numbers is how I have called this book, since I arrived at the methods, which deliver in it proofs of arithmetic theorems, through spatial considerations.

Ratner's work is a remarkable contribution in the general theme of applying "analysis of the infinite" and "spatial considerations" to number theory.

So what did Ratner prove in these remarkable papers? Perhaps the easiest to explain is her Orbit Closure Classification Theorem, confirming an important conjecture of M. S. Raghunathan:

Theorem 1 (Ratner's Orbit Closure Theorem [M3]). Let $G$ be a real linear algebraic group as above, $\Gamma$ a lattice in $G$ and $U<G$ a connected unipotent group. Then for any point $x \in G / \Gamma$ the closure of its $U$-orbit is a very nice object: a single orbit of some closed connected group $L$ that is sandwiched between $U$ and $G$ (and may coincide with either). Moreover, this single orbit of $L$ has finite volume.

Recall that the $U$-orbit of a point $x$ is simply the set $\{u . x: u \in U\}$. Note that in particular this shows that any $U$-orbit closure has a natural $U$-invariant probability measure attached to it. We also remark that one can loosen the requirement that $U$ be unipotent to $U$ being generated by one-parameter unipotent groups - the passage from Theorem 1 to this more general statement is not very difficult. Unlike previous work towards Raghunathan's Conjecture, in particular Margulis' proof in the mid 1980s of the (then) fifty year old Oppenheim Conjecture using a special case of Raghunathan's Conjecture, Ratner's route to classifying orbit closures was not direct but by via a measure classification result:

Theorem 2 (Ratner's Measure Classification Theorem $[\mathrm{M} 2, \mathrm{M} 1])$. Let $G, \Gamma$ and $U$ be as in Theorem 1. Then the only (Borel) probability measures on $G / \Gamma$ that are invariant and ergodic under $U$ are the natural measures on the orbit closures described in Theorem 1.

This requires a bit of explanation: We equip $X=G / \Gamma$ with the Borel $\sigma$-algebra $\mathcal{B}$, and consider probability measures on the measurable space $(X, \mathcal{B})$. Such a measure $\mu$ is $U$-invariant if the push forward of it under left multiplication by every $u \in U$ remains the same; $\mu$ is $U$-ergodic if every $U$-invariant Borel subset of $X$ is either null or conull. Every $U$-invariant probability measure can be presented as an average of ergodic ones, hence classifying the $U$-ergodic measures gives a description of all $U$-invariant probability measures on $X$. Dani conjectured this measure classification result in the same paper where Raghunathan's Conjecture first appeared.

It is possible to reduce both Theorem 1 and Theorem 2 to the case where $U$ is a one-parameter unipotent group. The following theorem implies both of the theorems quoted 
above in the one-parameter case, but is used by Ratner as a bridge allowing her to pass from the measure classification theorem (which, as we said in the outset, is the heart of her work on unipotent flows) to the obit closure theorem:

Theorem 3 (Ratner's Distribution Rigidity Theorem [M3]). Let $G, \Gamma, U$ be as above, and let $x \in G / \Gamma$. Then there is a $U$-ergodic probability measure $m_{x}$ of the form given above (i.e. the uniform measure on a finite volume orbit of a connected group sandwiched between $U$ and $G$ ) so that $X$ is in the support of $m_{x}$ and for any bounded continuous function $f$ on $G / \Gamma$ we have that the ergodic averages

$$
\frac{1}{T} \int_{0}^{T} f(u(t) \cdot x) d t \rightarrow \int f d m_{x} \quad \text { as } T \rightarrow \infty .
$$

The reader with some basic knowledge of ergodic theory might be fooled to think that (0.1) is an application of the Birkhoff Pointwise Ergodic Theorem. Not so! The Birkhoff Pointwise Ergodic Theorem only gives information about almost every point (with respect to a given ergodic measure). The whole point of Ratner's Distribution Rigidity Theorem is that it is true for each and every $x \in G / \Gamma$. Almost everywhere results are almost always much easier to prove, ${ }^{2}$ but in a mathematical manifestation of Murphy's Law, such results might say something about virtually all points but if you are given a specific point and want to study its behaviour under a given action they tell you absolutely nothing. To give a simple analogy, it is trivial to prove that for a.e. $x \in[0,1]$ the asymptotic density of occurrence of each of the digits $0,1,2, \ldots, 9$ in the decimal expansion of $x$ is $1 / 10$, but asking whether this holds for particular numbers of interest such as $2^{1 / 3}$ or $\pi$ seems at present to be a hopelessly difficult question!

As it turns out, for some of the most juicy applications of these rigidity results a more general setup is required. To begin with, one may consider linear algebraic groups over other fields; and since the topological structure is very much in play here, the natural class of fields to look at are local fields, i.e. topological fields whose topology is locally compact, such as $\mathbb{R}$ or the $p$-adic numbers $\mathbb{Q}_{p}$. Both Ratner [M4] and independently Margulis and Tomanov [GAGM] extended the above results to this setting, and more generally to quotients $G / \Gamma$ where $G=\prod_{i=1}^{k} G_{i}$ with each $G_{i}$ a linear algebraic group over a local field of characteristic zero. ${ }^{3}$ We shall refer to such quotient spaces $G / \Gamma$ as $S$-arithmetic quotients, a terminology that probably needs some explanation which we omit to avoid too much of a

\footnotetext{
${ }^{2}$ This is a slight pun- "almost everywhere" is used in the above sentence in its precise mathematical sense, whereas "almost always" is used in the ordinary, non-mathematical sense of the phrase..

${ }^{3}$ Note that our definitions of unipotent groups and one-parameter unipotent groups make sense over any field, and can be easily extended to the product case, e.g. a subgroup $U<$ $\prod_{i=1}^{k} G_{i}$ (with each $G_{i}$ defined over a different local field) is a one-parameter unipotent group if there is an $i$ so that $U$ is a one-parameter unipotent subgroup of $G_{i}$.
}

digression. It would have been interesting to have such rigidity results also for local fields of positive characteristic such as $\mathbb{F}_{q}((t))$ - the field of formal Laurent series with coefficients in the finite field $\mathbb{F}_{q}$ with $q$ elements - but there seem to be serious technical obstacles to doing so and only partial results in this direction are known.

The rigidity theorems of Ratner have had numerous applications in many areas of mathematics. A highly nontrivial special case of her general measure classification result, namely the classification of measures on a reducible product $\left(\mathrm{SL}(2, \mathbb{R}) / \Gamma_{1}\right) \times\left(\mathrm{SL}(2, \mathbb{R}) / \Gamma_{1}\right)$ invariant under a one-parameter unipotent group (the interesting case is classifying measures that project to the uniform measure on each $\left(\operatorname{SL}(2, \mathbb{R}) / \Gamma_{i}\right)$ factor, or in the ergodic theoretic terminology, joinings) was proved by Ratner already in the early 1980s. The original motivation of Ratner in studying these flows was to understand better (and give natural examples for) a property of measure preserving systems called Loosely Bernoulli - we can view this somewhat anachronistically as an application of unipotent flows to the abstract theory of dynamical systems. Since then her work has had several other applications to abstract ergodic theory and descriptive set theory. There are very striking applications of her work to mathematical physics, for instance in the work of Marklof and Strömbergsson on the Lorentz gas, and to geometry. In this note we have chosen to highlight a couple of the many applications of her theorems (as well as the extension to products of linear groups over local fields as above) to number theory.

In making his famous conjecture, Raghunathan was motivated by the connection to the Oppenheim Conjecture, a connection that allowed Margulis to resolve this longstanding open problem by establishing a special case of the conjecture posed by Raghunathan [G]. Oppenheim conjectured in the 1930s that for any indefinite quadratic form $Q$ in $d \geq 3$ variables that is not proportional to a quadratic form with integer coefficients, the set of values attained by $Q$ at integer vectors, that is to say $Q\left(\mathbb{Z}^{d}\right)$, contains zero as a non-isolated point. Using Ratner's Measure Classification Theorem, and relying upon prior work by Dani and Margulis, Eskin, Margulis, and Mozes [AGS] were able not only to show that there are integer vectors $\mathbf{n} \in \mathbb{Z}^{d}$ for which $Q(\mathbf{n})$ is close to a given value (say 0 ), but to count the number of such vectors. More precisely, for indefinite quadratic forms as above, not of signature $(1,2)$ or $(2,2)$, Eskin, Margulis, and Mozes show that for any $a<b$, the number of integer vectors $\mathbf{n} \in \mathbb{Z}^{d}$ inside a ball of radius $R$ for which $a<Q(\mathbf{n})<b$ is asymptotically given by the volume of the corresponding shape cut by the two hypersurfaces $Q(\mathbf{x})=a$ and $Q(\mathbf{x})=b$ in this ball. Perhaps an illustration of the delicacy of the question is that this natural statement is false(!) for quadratic forms of signature 
$(1,2)$ or $(2,2)$, though in a follow-up paper Eskin, Mozes, and Margulis were able to prove this estimate for quadratic forms of signature $(2,2)$ under a suitable Diophantine condition, a result which is of interest in the context of the study of the statistics of energy levels of quantization of integrable dynamical systems.

The reason unipotent dynamics is relevant to the Oppenheim Conjecture is that the symmetry group of an indefinite (real) quadratic form with $\geq 3$ variables contains (indeed, is generated by) one-parameter unipotent groups. Surprisingly, there is a relatively recent application of the S-arithmetic analogue of Ratner's results to positive definite, integral forms.

Legendre's Three Squares Theorem says that a positive integer $n$ can be presented as a sum of three squares if and only if it is not of the form $4^{a}(8 b+7)$, with $a, b$ integers. This is an example of a local-to-global principle: the quadratic form $Q(x, y, z)=x^{2}+y^{2}+z^{2}$ represents an integer $n$ if and only if the congruences $Q(x, y, z) \equiv n$ $\left(\bmod p^{a}\right)$ are solvable for any prime $p$ and any $a \in \mathbb{N}$ (for a given $p$, consistency of this infinite set of congruences is equivalent to $Q(x, y, z)=n$ being solvable by $p$-adic integers). In this particular case, only the prime 2 can be an obstacle though there is another restriction on $n$ implicit in the way that we set up the problem that $n$ is positive - which can be said to come from the "place at infinity," in other words from the necessity that $Q(x, y, z)=n$ be solvable over $\mathbb{R}$.

Legendre's Three Squares Theorem can be viewed as a special case of the following problem: Given a fixed positive definite integral quadratic form $Q$ in many (say $k$ ) variables, which quadratic forms $Q^{\prime}$ in $\ell<k$ variables can be represented by $Q$ ? That is to say, when can we find a $k \times \ell$ integer matrix $M$ so that as quadratic forms $Q^{\prime}=Q \circ M$ ? For $\ell=1$ and $Q=x^{2}+y^{2}+z^{2}$ this reduces to the question addressed by Legendre: the form $Q^{\prime}=n x^{2}$ can be represented by $Q$ iff $n$ can be written as a sum of three squares. Local solvability - the existence of such matrix $M$ with entries in $\mathbb{Z}_{p}$ for every $p-$ is an obvious necessary condition that can be verified with a finite calculation.

Hsia, Kitaoka, and Kneser in 1978 established the validity of such a local-to-global principle for representing any form $Q^{\prime}$ in $\ell$ variables with sufficiently large square free discriminant by a given form $Q$ in $k$ variables once $k \geq 2 \ell+3$ by using more traditional number theoretic methods. This remained the best result on this very classical problem (essentially dating back to the work of Gauss) until Ellenberg and Venkatesh [JA] were able to use the $S$ arithmetic extensions to Ratner's Orbit Closure Theorem to very significantly reduce the restriction on $k$ and $\ell$ to be $k \geq \ell+5$. While we cannot get into the details of the argument, we note that even if a quadratic form $Q$ is positive definite, hence its symmetry group over $\mathbb{R}$ is compact, over the $p$-adic numbers in general for $k \geq 3$ variables it would be a non-compact group with plenty of unipotents. In truth, the relevant symmetry group for this case is not the symmetry group of $Q$ but the subgroup of this symmetry group fixing a given quadratic form in $\ell$ variables, but this is precisely why in this problem one needs to employ $p$-adics.

An even more surprising application of Ratner's work to number theory was given by Vatsal and Cornut-Vatsal (e.g. $[\mathrm{V}]$ ). We do not give details here, but in these works families of elliptic curve $L$-functions, and in particular their central values (or derivatives thereof when their functional equation is odd rather than even), are considered. Using Ratner's Orbit Closure Theorem as a basic ingredient Vatsal (and in the more general cases Cornut and Vatsal) showed that all but finitely many of these values are not zero. When combined with well-known results towards the Birch and Swinnerton-Dyer Conjecture, this proves a conjecture of Mazur: essentially all the points on an elliptic curve whose coordinates lie in ring class fields with restricted ramification are generated by explicit special points first constructed by Heegner.

The impact of Ratner's work cannot be measured only by direct application of her seminal results. Techniques introduced by Ratner to study ergodic theoretic joinings in her early works on unipotent flows in the 1980s were a main inspiration in the work of the first named author on diagonalizable flows and its applications to Arithmetic Quantum Unique Ergodicity and equidistribution. Benoist and Quint were similarly inspired by Ratner's work in their breakthrough work understanding stationary measures and orbit closures for actions of thin groups on homogeneous spaces, and Eskin and Mirzakhani transformed the study of moduli spaces of abelian and quadratic differentials on Riemann surfaces by proving an analogue of Ratner's work in this setting.

The prevalence of deep and suprising applications of Ratner's Rigidity Theorems on unipotent flows is remarkable, and shows the richness of the subject of homogeneous dynamics and how interconnected it is with many other subjects. It is also a tribute to a wonderful mathematician who has left a legacy to future mathematicians for many years to come.

\section{References}

[AGS] Eskin A, Margulis G, Mozes S. Upper bounds and asymptotics in a quantitative version of the Oppenheim conjecture, Ann. of Math. (2), no. 1 (147):93-141, 1998, DOI 10.2307/120984. MR1609447 
[JA] Ellenberg J, Venkatesh A. Local-global principles for representations of quadratic forms, Invent. Math., no. 2 (171):257-279, 2008, DOI 10.1007/s00222-007-0077-7. MR2367020

[G] Margulis G. Discrete subgroups and ergodic theory, Number theory, trace formulas and discrete groups (Oslo, 1987); 1989:377-398. MR993328

[GAGM] Margulis G A, Tomanov G M. Invariant measures for actions of unipotent groups over local fields on homogeneous spaces, Invent. Math., no. 1-3 (116):347-392, 1994, DOI 10.1007/BF01231565. MR1253197

[M1] Ratner M. On measure rigidity of unipotent subgroups of semisimple groups, Acta Math., no. 3-4 (165):229-309, 1990, DOI 10.1007/BF02391906. MR1075042

[M2] Ratner M. On Raghunathan's measure conjecture, Ann. of Math. (2), no. 3 (134):545-607, 1991. MR1135878

[M3] Ratner M. Raghunathan's topological conjecture and distributions of unipotent flows, Duke Math. J., no. 1 (63):235-280, 1991, DOI 10.1215/S0012-7094-91-063118. MR1106945

[M4] Ratner M. Raghunathan's conjectures for Cartesian products of real and $p$-adic Lie groups, Duke Math. J., no. 2 (77):275-382, 1995, DOI 10.1215/S0012-7094-95-077102. MR1321062

[V] Vatsal V. Uniform distribution of Heegner points, Invent. Math., no. 1 (148):1-46, 2002, DOI 10.1007/s002220100183. MR1892842

\section{Credits}

Photo of Marina Ratner is courtesy of Anna Ratner. 


\section{To the Memory of Marina Ratner}

\section{Yakov Sinai}

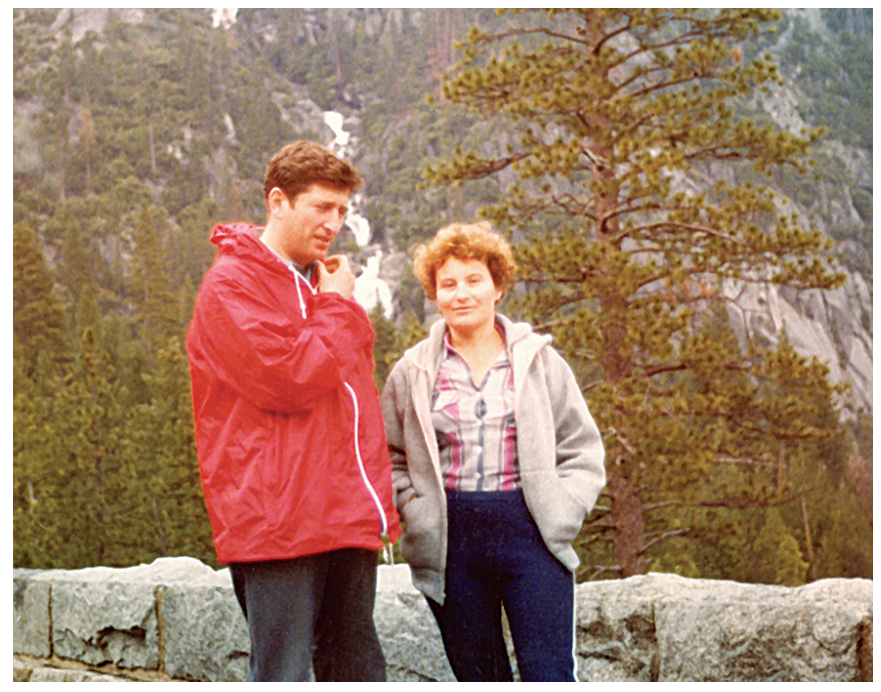

Sinai and Ratner in 1978.

I met Marina almost the same time as her elder sister, Yulia. Their father was a famous biologist. Marina started as a student of Moscow State University, and initially she was a student of A. N. Kolmogorov and later became a student of R. L. Dobrushin. When she entered graduate school she became interested in ergodic theory. This is how I became her advisor.

Marina married A. Samoilov when they both were undergraduate students of the second year. Their marriage didn't last long and soon they separated, though they maintained contact. Marina was left with her daughter, Anya. Marina was very close to the family of her daughter. During many years, Marina spent a lot of time with her grandchildren. Marina's friends knew that it was strictly forbidden to call her on Saturdays because she was always busy working the whole day with her grandson.

Yakov Sinai is a professor of mathematics at Princeton University. His email address is sinai@math. princeton . edu.

For permission to reprint this article, please contact: reprint -permission@ams .org.

DOI: http://dx.doi.org/10.1090/noti1830
Marina wrote her thesis about Markov partitions in multi-dimensional systems. At that time it was a very hot topic. One of the referees of Marina's thesis was V. A. Rokhlin, who wrote a very good report. This was important because the Scientific Council where Marina's thesis was considered was known at that time for its antisemitism. It was rather surprising that in the case of Marina the system worked well, including the voting of the PhD committee and the approval of the High Attestation Committee (VAK).

Quite soon she started to work in one of the Moscow Institutes where Marina was appointed to her first academic position. The fact that she got a position so quickly was rather unusual at the time.

In another case it would be a big step in someone's career. But not for Marina, because quite soon she decided to go in a different direction and applied to emigrate to Israel together with her daughter.

In Israel she joined the Institute of Mathematics at the Hebrew University and started teaching there. Marina did everything very well. Soon she became famous among her students. Many of them kept as souvenirs the notes left after Marina's classes.

A bit later Marina heard about some vacancies opening in Berkeley and moved there with her family. Berkeley became her home until the end of her life. She was elected as a full member of the National Academy of Sciences of the USA, and was invited as a plenary speaker to ICM-94 in Zürich.

Marina had many close friends in Berkeley and other places. One can mention Smale, Ornstein, Arnold, Fuchs, Pyatetskii-Shapiro, Kazhdan, Chorin, Zalenko, Kresin, and many others. Marina was always ready to help her friends and other people. I remember the case when our family arrived to Princeton after my son had seriously broken his leg. Marina contacted many people and eventually they helped us to find V. Golyakhowsky, who was a remarkable orthopedist. His treatment was excellent, and my son completely recovered and can freely walk now without any trace of the previous accident. 
Marina was involved in many types of social and political activity, and was very strong and principled in promoting the causes that she believed in. For instance, she had strong opinions about mathematical education and education in general.

This text is a small part of what can be written about Marina. She was a great mathematician, a remarkable personality, and a close friend.

\section{Credits}

Photo is courtesy of Anna Ratner. 


\section{Meetings: For Almost All of Our Lives}

\section{Boris Gurevich}

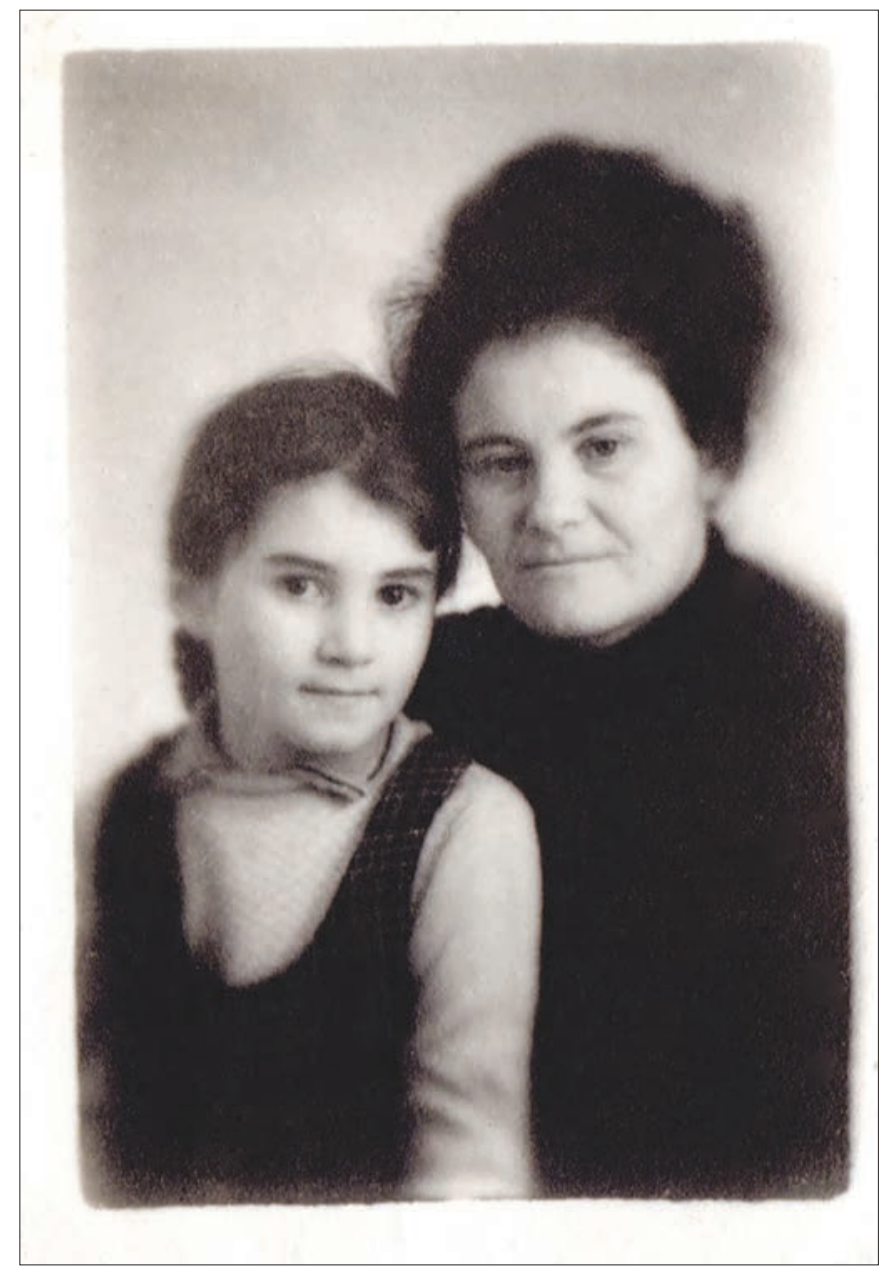

Ratner with her daughter Anna, 1971.

Boris Gurevich is a professor of mathematics at Lomonosov Moscow State University. His email address is gurevich@mech.math.msu.su.

For permission to reprint this article, please contact: reprint -permission@ams.org.

DOI: http://dx.doi.org/10.1090/noti1831

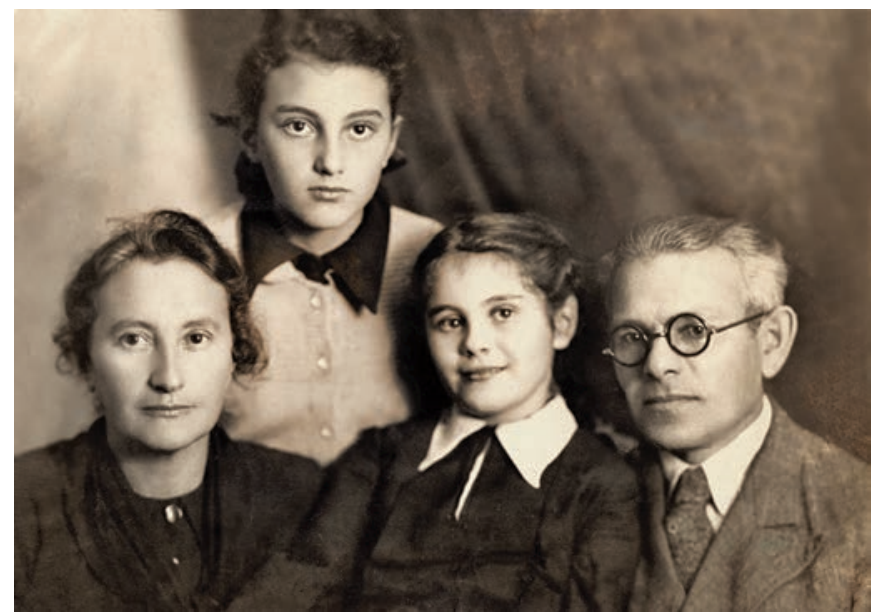

Ratner with her family, 1947 (left to right): mother Ksia, sister Yulia, Marina, and father Yehoshua.

I cannot claim that I have been Marina's close friend from our very first meeting. But I believe at some moment this became so. Our meetings lasted for many years, with frequency dependent on circumstances of our lives and on political events as well. I am going to remember several of these meetings in the hope that my story will shed additional light on this remarkable character.

I got to know Marina when we were about seven years old and went to the same musical school for children. By coincidence we had the same piano teacher, whose name was Anna Ratner. In one or two years I moved to another musical school, closer to my home, and we lost one another for several years.

Our next meeting occurred at the Mechanics and Mathematics Department of Moscow State University, where we entered simultaneously and quite independently. I happened to meet Marina at one of the first lectures and recognized her almost immediately, strange though it may seem. That is why when in several days our very sociable 


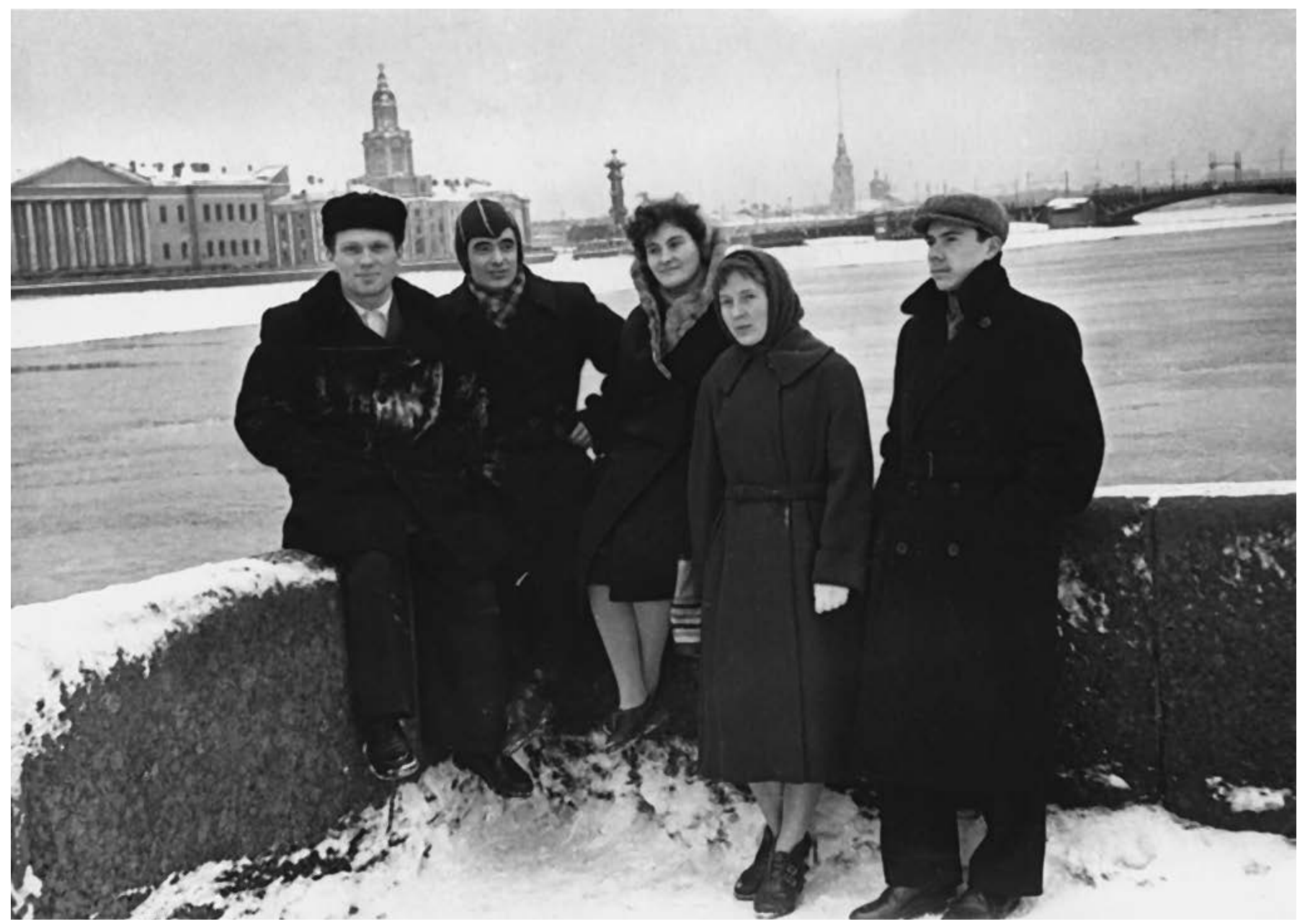

Visiting Leningrad with fellow students - (left to right) Ilya Mindilin, Vener Galin, Lena Odnorobova (orVera Steniushkina), Slava Perlov) -from Moscow, circa 1961.

fellow student tried to introduce me to her, this sounded funny for both of us.

During the first two years we were in different groups and met only at common lectures. But in our third year, everybody had to choose a specialization. And again, we made the same choice, which was probability, and got into the same group.

At that time the probability and statistics subdivision of the department was headed by A. N. Kolmogorov, and almost all who worked there were his former students. Kolmogorov was very active in various directions; in a few years he included Marina in a small, young team involved in his study of statistical laws in language. But her first supervisor was R. L. Dobrushin, who, as I know, liked very much her master's thesis in information theory.

Upon graduating from the university, Marina was for some time working at Kolmogorov's boarding school, a high school for gifted children from all over the country founded by Kolmogorov and later named after him. She also took part in the preparation of the principal works of Claude Shannon for publication in Russian.
About that time she married a student from our course, and I met her not too often. But in 1965 she came back to the university as a graduate student under the supervision of Y. G. Sinai, and our meetings became regular again because we both attended seminars on ergodic theory.

Once we examined an undergraduate. I began with a question, then Marina entered and I went out for some time, while she continued. When I came back, I was not pleased that she finished too fast. But later I decided that she was right: first, this student was Lenya Bunimovich, and second, Marina was very thorough in everything. Once, already in Berkeley, she observed that in one of her papers, it was written "weak convergence" instead of "weak* convergence," and she asked me to insert the asterisk by pen each time I was at a library where a journal with the paper was accessible. She told me, "I am a perfectionist," and this was the truth.

She published several papers and defended her PhD thesis on geodesic flows in 1969. Thereafter she was teaching at one of the technical universities in Moscow, but not for very long, because she applied for emigration to Israel. 


\section{MEMORIAL TRIBUTE}

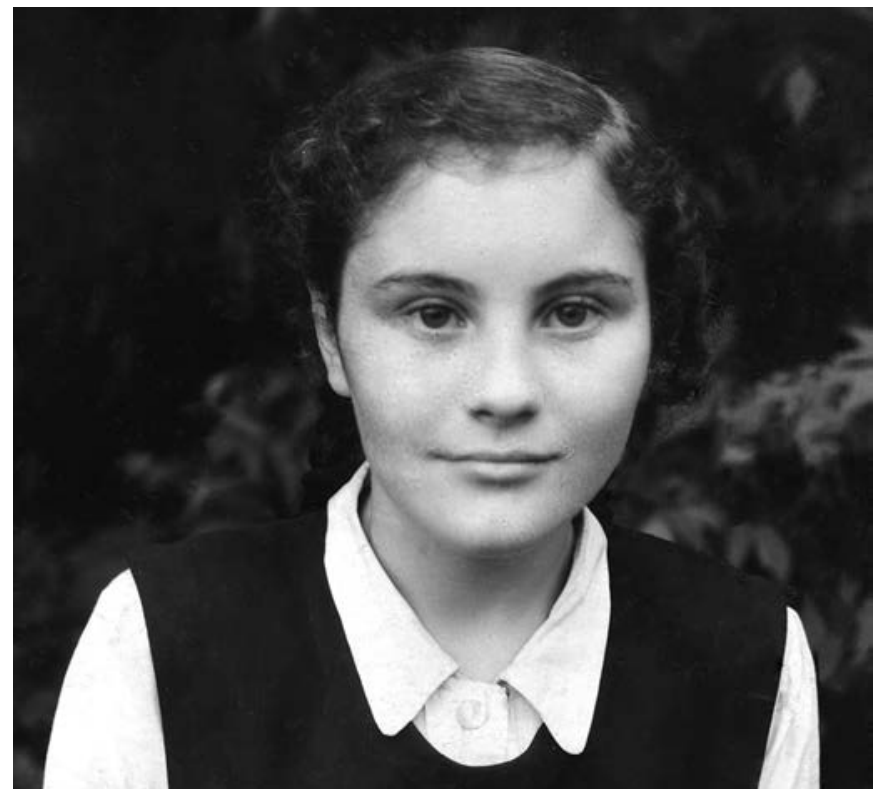

As a girl of twelve, 1950 .

Honestly speaking, I initially considered her intention reckless: I knew that she was going alone, with a small daughter, without language and having no relatives there.

But I had underestimated Marina: she overcame difficulties, which were indeed considerable, and in 1971 she was already working at the Hebrew University. At the time, the Soviet Union had no diplomatic relations with Israel and postal services were unreliable. Of fragmentary information from Marina I remember that in the fall of 1973, the university professors were asked to write their lectures down in order that the students, turned into soldiers for a while, could read them at the front.

When Marina moved to Berkeley, I used a possibility to hear something of her from J. Feldman, whom I met in 1977 in Warsaw at a conference on ergodic theory.

Only when Gorbachev came to power did mutual visits become possible. Marina came to Moscow more than one time in the 1990s and early 2000s. Once she left for a few days for Minsk, where a mathematical conference was conducted. Being aware of food shortage in Moscow at the time, she bought in Minsk, on her own initiative, some cheese for a small child of our friend. I appreciated her solicitous concern for her friends once again when I visited her at Berkeley in the late 1990s.

As far as I know, she came to Moscow for the last time in June of 2003 to the conference devoted to Kolmogorov's centennial, where she was an invited speaker and met many old friends.

I saw Marina for the last time in May 2014 in Oslo, where we were invited by Sinai, our common teacher, as his guests as he was awarded the Abel Prize. We walked through the city in full lilac bloom and followed Sinai, visiting the town of Stawanger for one day, where we took, together with a few friends, an excursion along a fjord; Marina took a number of snapshots there.

She was always worried about the health of others. Throughout several years she insisted that I should regularly inform her of my state of health (results of tests, etc.). Answering my questions about her own health, she would always insist that she was splendidly sound. I have kept her last message of July 1, 2017, in which she wrote about her problems, but hoped that the treatment would eventually help. I also thought so.

\section{Credits}

All photos are courtesy of Anna Ratner. 


\section{In Memory}

\section{Ornstein}

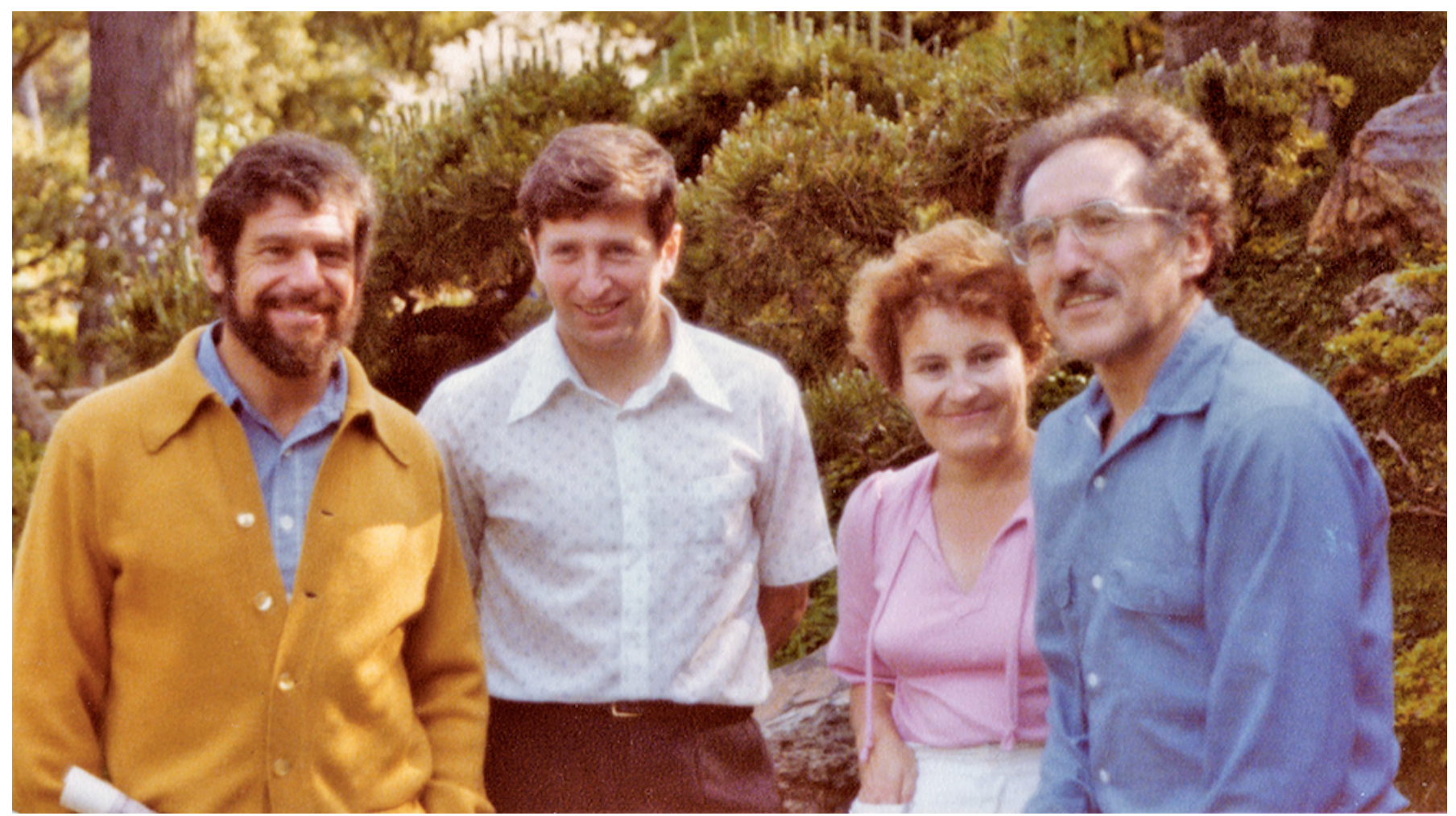

Ratner with (left to right) Ornstein, Yakov Sinai, and Jack Feldman, 1978.

Marina Ratner was a good friend and colleague. Although we never wrote a joint paper, we did work together, and I was able to gain great appreciation for the depth of her mathematical ability. The commitment to her family was very impressive; she homeschooled her grandchildren. She was not a fan of affirmative action and made it very clear that she wanted her achievements to be rated solely on her mathematics not her gender.

I would like to call attention to Marina's work on the horocycle flow, which I hope will not be overlooked in the

Donald Ornstein is a professor emeritus of mathematics at Stanford University. His email address is ornstein@math. stanford. edu.

For permission to reprint this article, please contact: reprint -permission@ams.org.

DOI: http://dx.doi.org/10.1090/noti1832 light of her later and more spectacular results. The study of the horocycle flow and the geodesic flow as flows on an abstract measure space began in 1938 with the work of Hedlund and Hopf. While the geodesic flow is the most random measure preserving flow on an abstract measure space, the horocycle flow has the opposite behavior. Marina elucidated its rigidity properties.

I will give just one example. If two horocycle flows on the quotient space $M$ of $\operatorname{SL}(2, \mathbb{R})$ by a discrete subgroup are the same as measure preserving flows, then the underlying surfaces are conformally isometric. This means that even though we replaced $M$ by an abstract measure space, the flow retains all of the geometry that we threw out.

\section{Credits}

Photo is courtesy of Anna Ratner. 


\section{Marina Ratner, quelques évocations}

\section{Jean-Paul Thouvenot}

At my first encounter with Marina, in Jerusalem, in 1974, just after the Lavi Conference, I saw a young woman quite shy, sweet and smiling, pleased to receive a gift that a common friend had prepared for her from Paris. I met her later in Berkeley when she was already settled; I paid several visits there. Memory is sometimes strangely selective-I remember distinctly that, in an excursion which we took together with her and the Katok family to "Pebble Beach" (this I am not sure of), she had a very battered car, with the exhaust threatening to fall off at every turn.

Her mathematics, which had started in Moscow with Sinai, received the influence of the California environment, and one of her first works there was the proof that the horocycle flow is loosely Bernoulli, an abstract measure-theoretic property that was quite popular at that time in Berkeley, the impetus for it having been given by Jack Feldman. A second paper, which came quite quickly, was that the Cartesian square of the horocycle flow is not loosely Bernoulli. This was, for the group of people working in this field, quite unexpected and very strong. The nonloosely Bernoulli property all of a sudden being attached to a simple algebraic object, while all previous examples, starting with the one of Jack Feldman, required elaborate combinatorial constructions. This work of Marina is extremely difficult to read, and I remember, when I came to complain (the last time was not so long ago) to her, "But it is so simple, just follow what is written, everything is completely natural, you will not find any obstacle..." In this same work appeared for the first time the "shearing" that was going to play such an important role in her subsequent works. And then came, in an extraordinary succes-

Jean-Paul Thouvenot is directeur de recherches émérite au CNRS à Sorbonne Université. His email address is jean-pau1. thouvenot Qupmc.fr.

For permission to reprint this article, please contact: reprint -permission@ams .org.

DOI: http://dx.doi.org/10.1090/noti1833 sion across a few years of mathematical excitement, a list of papers in which she developed her theory of horocycle flows culminating in the complete description of their joinings, which entails all their rigidity properties. Strikingly, Marina always worked alone and never had coauthors. As to joinings, she managed all by herself, and to my knowledge, without trying to get too many contacts with the people close to her who were active in this field at that time.

On a visit that she paid to Paris at about the same epoch, I put her up in a nice hotel close to Jussieu (as I usually did with visitors). But almost immediately, she came to me quite pleased to have moved with her daughter to a very modest place close to Gare de l'Est, proudly announcing, "Believe me, it is the best possible place as a starting point to visit Paris." Singular Marina!

Her work took a new turning point when she got, as an elaboration of her previous ideas, her fundamental result on the Ragunathan measure conjecture. Strangely, her mathematics, so deep, which is so much alive nowadays, and in so many different directions, is most frequently used as a black box or as a model.

It was a great shock to receive the message from $\mathrm{B}$. Weiss that she had died, shortly before a conference dedicated to the memory of Rufus Bowen, which she had accepted to attend.

I want to mention another memory, or more precisely, an image, because of the deep impression that it has left on me, although I cannot trace back exactly when it took place. I think that it was at a conference in Warwick: she was lecturing, so strong, so determined, and in appearance so fragile, all alone, in front of a huge audience.

In the same way as her mathematics does for our community, her presence, in the minds of those who have known her, persists with all the strength, the singularity, and the seduction of the exceptional. 


\section{Remembering Marina Ratner}

\section{$\mathrm{Hee} \mathrm{Oh}$}

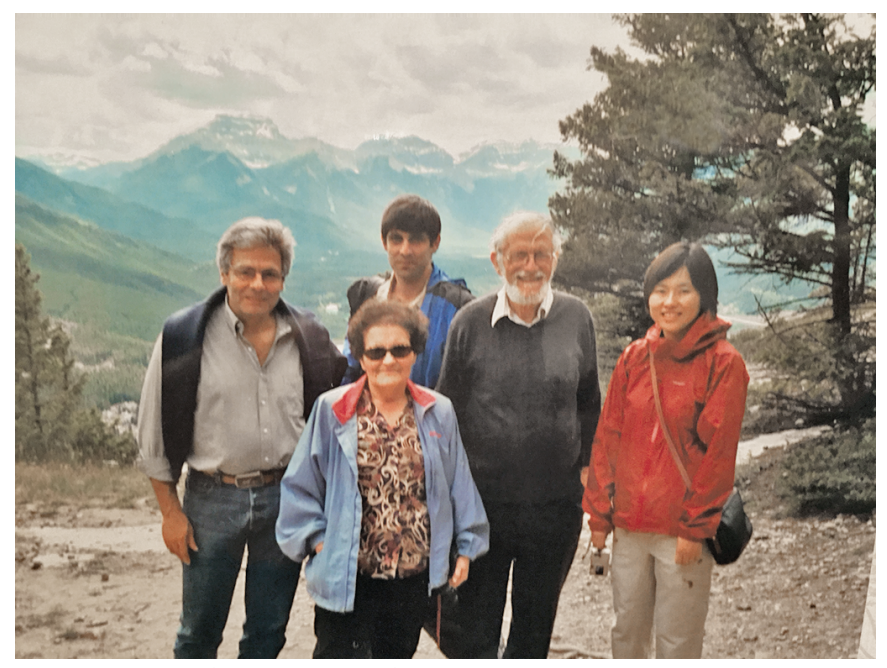

Ratner with (left to right) François Ledrappier, Dmitry Kleinbock, Hillel Furstenberg, and Oh, 2005.

I first met Marina at the International Conference on Lie Groups and Ergodic Theory held at TIFR in Mumbai in January of 1996. I was a fourth-year graduate student working with Gregory Margulis.

She gave a talk on the p-adic and $S$-arithmetic generalizations of her earlier proof of Raghunathan's conjecture. I remember how she began her talk with the assertion that while some notations and definitions may be standard, she still needed them to know for herself what she was talking about. She then went on to spend quite a big chunk of her time introducing a long list of notations and basic definitions, such as Ad-unipotents and $p$-adic Lie algebras. At the time, her talk was too technical for me to follow, but her uncompromising style left a strong impression on me.

My own lecture was about my ongoing thesis work on the arithmeticity of discrete subgroups in a higher rank simple Lie group generated by lattices in a pair of

Hee Oh is Abraham Robinson Professor of Mathematics at Yale University. Her email address is hee. oh@yale. edu.

For permission to reprint this article, please contact: reprint -permission@ams .org.

DOI: http://dx.doi.org/10.1090/noti1834 opposite horospherical subgroups, which was a conjecture of Margulis based on Selberg's earlier work in the case of a product of $S L(2, \mathbb{R})$ s. I had solved this conjecture for discrete subgroups of $\operatorname{SL}(n, \mathbb{R})$ for $n \geq 4$. Ratner's theorem on orbit closures was a key ingredient of my proof. I did not get to receive any comments from her either on my talk or on my work at that time.

Seventeen years later in 2013, there was a conference in her honor titled "Homogenous Dynamics, Unipotent Flows, and Applications" at the Hebrew University. I had just finished my joint work with Amir Mohammadi on the classification of joining measures for geometrically finite subgroups of $\operatorname{SL}(2, \mathbb{R})$ or of $\operatorname{SL}(2, \mathbb{C})$. It was an extension of her work "Horocycle flows, joining and rigidity of products," published in Annals of Mathematics 1983, and our approach was to adapt her proof in the infinite-volume setting. I opened my lecture saying that I was proud of my mathematical aunt; she and Margulis shared a common advisor, Sinai. I then successfully squeezed the two subjects of discrete groups and joinings into my one-hour lecture and closed with the statement that I had started my mathematical career by applying Ratner's theorem as a black box and that I was now hoping to generalize her ideas in the infinite-volume setting. After my lecture, I asked Marina directly, "Did you like my lecture?" She said, "Yes, very much," with a big emphasis on "very," and asked, "Why don't you post your lecture notes in your webpage?" I jokingly replied to her, "Marina, who is going to read it?"

She once said in an email to me, "If a woman is good in math, she does not need encouragement or a role model. I remember when I was young, no matter what anyone would say, I knew that I would go to math. I did not need any encouragement for that. The same is probably true about you. Did you need encouragement?"

I wrote back, saying, "Marina, whether you wanted to or not, you have been a great source of pride and inspiration for female mathematicians in the area. I am very grateful to you for having been such a great role model."

Thank you Marina.

\section{Credits}

Photo is courtesy of Nimish A. Shah. 